\title{
Editorial \\ Nitrogen-Containing Molecules: Natural and Synthetic Products including Coordination Compounds
}

\author{
Dimitris Matiadis *(D) and Eleftherios Halevas *(D) \\ Institute of Biosciences and Applications, National Centre for Scientific Research "Demokritos", \\ 15310 Athens, Greece \\ * Correspondence: matiadis@bio.demokritos.gr or dmatiadis@gmail.com (D.M.); \\ lefterishalevas@gmail.com (E.H.); Tel.: +30-210-650-3558 (D.M. \& E.H.)
}

check for updates

Citation: Matiadis, D.; Halevas, E. Nitrogen-Containing Molecules: Natural and Synthetic Products including Coordination Compounds. Molbank 2021, 2021, M1282. https://doi.org/10.3390/M1282

Received: 7 September 2021

Accepted: 24 September 2021

Published: 27 September 2021

Publisher's Note: MDPI stays neutral with regard to jurisdictional claims in published maps and institutional affiliations.

Copyright: (c) 2021 by the authors. Licensee MDPI, Basel, Switzerland. This article is an open access article distributed under the terms and conditions of the Creative Commons Attribution (CC BY) license (https:// creativecommons.org/licenses/by/ $4.0 /)$.
Nitrogen constitutes one of the most crucial elements in synthetic compounds, both in organic and in coordination chemistry. After all, urea is considered to be the first synthetic organic compound (it was first prepared in 1828 by Friedrich Wöhler from a mixture of the inorganic compounds silver cyanate $(\mathrm{AgOCN})$ and ammonium chloride $\left(\mathrm{NH}_{4} \mathrm{Cl}\right)$ ).

From a medicinal chemistry viewpoint, nitrogen is a very common element in every major class of active pharmaceutical ingredients existing in heterocyclic and in acyclic molecules. Functional groups like amines, imines, nitriles, amides, and carbamates dominate the libraries of bioactive compounds, whereas the total number of unique drugs containing at least one nitrogen heterocycle among FDA-approved pharmaceuticals is 910 ( $84 \%$ among 1035 unique small-molecule drugs). The top five most frequent nitrogen heterocycles in this list are piperidine, pyridine, piperazine, $\beta$-lactam, and pyrrolidine [1,2].

Until today, the combined use of synthetic organic chemistry and coordination chemistry has generated a number of new and effective synthetic methods of high-yield product formation for important classes of coordination compounds of several nitrogen-containing ligands with numerous metal ions, giving rise to novel materials with exceptional physicochemical, biological, medicinal, catalytic, and optical properties.

The aim of this Special Issue in the Molbank journal was, in principle, to collect original research articles related to one or more of the following: design and synthesis; structural characterization by means of NMR; X-ray crystallography; or other preliminary but significant results including, but not limited to, biological evaluation, theoretical calculations, molecular mechanics, and computational studies, material or physicochemical properties of nitrogen-containing molecules, and coordination compounds of nitrogencontaining molecules.

It was launched in August 2019 and, after two deadline extensions, was closed for new submissions in August 2021. Until this deadline, 16 exceptional contributions were made by authors from all over the world. Most of the contributions were made from authors based in Europe (12), with America (2) and Asia (2) following. Of note, is the broad geographic distribution of published articles, based upon the countries of the corresponding authors. Four of the published articles had authorship from Greece, two from Cyprus, two from the USA, and one from Russia, Japan, France, Saudi Arabia, Portugal, UK, and Spain. The majority (13) of the published content was in the field of synthetic chemistry, including synthetic methodologies and studies on chemical reactions and reactivity, while two papers dealt with coordination chemistry and one with structural elucidation. In particular, contributions related to organic synthesis may be divided in the subfields of aromatic reactivity (2), reactivity of heterocycles (2), synthesis of heterocycles (6), and detailed routine synthesis of very important compounds (3). Two of these papers included biological evaluation experiments.

The first two contributions were made from Kalogirou and Koutentis from Cyprus, in the field of aromatic reactivity. Kalogirou and Koutentis contributed with two communications on the reactivity of chlorinated pyrimidines. In their first paper, they focused on 
the displacement of carbon- 2 or carbon- 6 of polychlorinated pyrimidines using DABCO reagent (1,4-diazabicyclo[2.2.2]octane) with yields up to 52\% [3]. The second publication demonstrates the synthesis of 2-cyanopyrimidines from the corresponding 2-thiomethyl pyrimidines starting from the 4,6-dichloro functionalized analogues. The synthetic route involves the "activation" of carbon-2 by displacing one or both chlorides with electrondonating methoxy groups [4].

Chronologically, the first article on reactivity of heterocycles was made by Fedorowicz and co-workers. The authors reported the synthesis of a bis-quinoline carboxylic acid derived from the corresponding isoxazolo-quinoline and dimethyl acetylenedicarboxylate (DMAD) under mild alkaline conditions. DFT calculations revealed that the product was more favorable than the double Michael addition isoxazolone product, which according to the proposed by the authors' mechanism, is formed as an intermediate [5].

In the second paper on this topic, the ring opening reaction of a bicyclic vinyl aziridine by an appropriate tetrazole-thiol was demonstrated by Fortunato et al. towards the synthesis of a novel tetrazole bearing cyclopentenol. The reaction occurred under mild conditions $\left(37^{\circ} \mathrm{C}\right.$, water) and according to authors' claims, in a regio- and stereospecific manner. The product can be readily further functionalized via its primary and secondary hydroxyl groups [6].

Arranged in order of publication date, the first contribution on the field of the synthesis of heterocyclic compounds or the cyclization towards to heterocyclic ring formation, was one of the two papers published by our group. In this study, a 4-ethoxycarbonylphenyl functionalized 2-pyrazoline was prepared, via the cyclization reaction between the corresponding bis(arylidene)acetone and ethyl hydrazinobenzoate in high yield and purity as evidenced by HPLC experiments. The compound was designed as an agent that would be active against resistant cancerous, since similar pyrazolines or pyrazole analogues have been reported in the literature or recent patents for this purpose $[7,8]$. However, though DNA thermal denaturation and DNA viscosity tests revealed that the compounds act as DNA intercalators, no activity against doxorubicin-resistant breast cancer or synergistic activity could be detected [9].

Konstantinova et al. reported the formation of a novel fused oxazinoxazine from ethyl 2-(hydroxyamino)propanoate and 2-aminophenol treated with $\mathrm{S}_{2} \mathrm{Cl}_{2}$. The structure of the product was also determined by $\mathrm{X}$-ray crystallographic analysis. It is worth mentioning that this compound was obtained unexpectedly, instead of a dithiazole derivative and that following the same procedure using ethyl 2-oxopropanoate, the reaction did not occur [10].

Starting from $\beta$-ketoester derivative and 2-aminopyridine, Tenti and co-workers prepared a novel functionalized imidazo[1,2-a]pyridine-2(3H)-one in two steps. The first step involved the formation of a $\beta$-ketoamide, which was isolated and characterized. Next, its bromination in dichloromethane, following a cyclization by intramolecular $\mathrm{S}_{\mathrm{N}} 2$ displacement of the intermediate, afforded the final nitrogen heterocycle. NMR experiments revealed that the enol isomer is predominant rather than the $\beta$-dicarbonyl analogue [11].

The synthesis of a spiro carbocyclic hydantoin with potential pharmacological interest was investigated by Pardali and co-workers in their contribution as a short note paper. The target molecule was obtained in three steps starting from 4-phenyl cyclohexanone. DFT calculations were performed in order to determine and study the most favorable structures [12].

The synthesis of a novel oxazolocoumarin incorporating an amine group was prepared by Vlachou et al. in three steps starting from 6-hydroxycoumarin. The first step involved the nitration of the starting material with cerium ammonium nitrate (CAN) resulting to the formation of three isolable compounds. The main product, 6-hydroxy-5,7-dinitrocoumarin, was cyclized with $p$-tolylmethanol via an $\mathrm{Au} / \mathrm{TiO}_{2}$ catalyzed reaction and subsequent reduction of the nitro group. The target compound was evaluated as possible antioxidant agent and inhibitor of soybean lipoxygenase with no or low activity [13].

The communication submitted by Ubeid et al. deals with the synthesis of a benzaldehyde compound derived from 2-mercaptobenzimidazole and its thiosemicarbazone 
analogue. The latter, which is a novel compound, was fully characterized and obtained in high purity and high yield (75\%). However, the highlight of this study was the report of an improved method for the synthesis of the intermediate benzimidazole-benzaldehyde conjugate. According to the literature, this compound and similar compounds have been synthesized with the use of copper(I) iodide or irradiation and long reaction times $(>12 \mathrm{~h})[14,15]$, whereas the reported procedure involves the addition of $\mathrm{K}_{2} \mathrm{CO}_{3}$ for $1 \mathrm{~h}$ under reflux with a near to quantitative yield [16].

The following three papers involve the routine synthesis of one or more compounds of remarkable interest. All compounds were fully characterized and their synthetic protocols were described in detail. Asquith and Tizzard demonstrated the preparation and characterization of a 4-anilinoquinoline derivative, incorporating a difluoromethyl group, with potential biological properties. The target molecule was synthesized by routine synthesis from the corresponding 4-chloroquinoline and 3-(difluoromethyl)aniline. The crystallographic analysis of its hydrochloric acid is reported as well [17].

Husson and Guyard presented the synthesis of the novel 4'-(5-N-propylthiophen2 -yl)-2, $2^{\prime}: 6^{\prime}, 2^{\prime \prime}$-terpyridine, with potential application as a ligand for the preparation of metal complexes. The compound was obtained by the reaction between 2-acetylpyridine and 5- $\mathrm{N}$-propylthiophene-2-carboxaldehyde, in high purity (>98\%), as determined by quantitative NMR [18].

Banks et al. reported the synthesis of ten polar aromatic compounds bearing an alkynyl imine or amide. The products were obtained from inexpensive and commercially available starting materials in good yields under mild reaction conditions. The study is supplemented with a three-step synthetic protocol for the preparation of a catechol containing an alkynyl amine. This route proceeded via the protected catechol as acetonide [19].

Because there are no structurally characterized metal complexes of Schiff bases derived from gamma-amino acids, our research group was motivated to develop a novel $\mathrm{Cu}$ (II) complex based on a new Schiff base obtained by the condensation of ortho-vanillin with gamma-aminobutyric acid. The novel compounds were prepared in good yields and purity and were chemically, spectroscopically and structurally characterized through elemental analysis, HR-ESI-MS, FT-IR, UV-Vis, NMR, and single crystal X-ray diffraction. The crystal structure of the produced $\mathrm{Cu}(\mathrm{II})$ complex reflects an one-dimensional polymeric compound. The $\mathrm{Cu}(\mathrm{II})$ ion is bound to two singly deprotonated Schiff base bridging ligands forming a $\mathrm{Cu}^{(\mathrm{II})} \mathrm{N}_{2} \mathrm{O}_{4}$ chelation environment, and a coordination sphere with a disordered octahedral geometry [20].

Takase et al. focused their interest on the development of transition metal complexes, particularly those in groups 7 and 8 , bearing non-innocent azopyridyl ligands including $2,2^{\prime}$-azopyridine (apy) and 2-phenylazopyridine (pap), that find application as multifunctional materials with impressive magnetic properties. They reported a newly synthesized diradical neutral dicarbonylruthenium(II) complex, bearing two azopyridyl ligands coordinated as anion radicals, by introducing in its structure two $\mathrm{CO}$ molecules as ligand and reducing agents. Further magnetic studies on the complex revealed paramagnetic and antiferromagnetic interactions between the spins in each radical [21].

Aitken et al. reported the successful crystallization and determination of the X-ray structure of homopiperazine, a well-known heterocyclic compound with remarkable properties as a component of liquids for $\mathrm{CO}_{2}$ capture and as a component of various organic and organic/inorganic supramolecular ionic salts and transition metal complexes. The X-ray studies revealed the pseudo-chair conformation of the molecule, with each $\mathrm{NH}$ acting both as a hydrogen-bonding donor and acceptor, leading to the formation of a complex network structure. Further NMR analysis facilitated the determination of the three one-bond $\mathrm{CH}$ coupling constants and the ${ }^{15} \mathrm{~N}$ chemical shift [22].

This Special Issue on "Nitrogen-Containing Molecules: Natural and Synthetic Products Including Coordination Compounds" was launched two years ago with enthusiasm from two early-career researchers, based in the National Centre for Scientific Research "Demokritos", in Athens, Greece, with different backgrounds and expertise; organic and 
medicinal chemistry (Dr. Dimitris Matiadis) and inorganic and bioinorganic chemistry (Dr. Eleftherios Halevas). We are thrilled that the Special Issue has exceeded all our expectations with the submission and publication of many exceptional contributions from scientists on the highest level from all over the world. We would like to express our sincere gratitude to all authors who contributed and chose our Special Issue to publish their work. We also wish to thank all the reviewers for the evaluation of the submitted articles and the Editorial Office of Molbank, especially Mrs. Jade Lu, for the fast and professional handling of the manuscripts and for addressing any issue that arose in these two years. The success of this Special Issue shows the ongoing importance of this topic in chemistry and encourages us to consider editing other Special Issues in this area in the near future.

Author Contributions: Writing—original draft preparation, D.M. and E.H.; writing-review and editing, D.M. and E.H. All authors have read and agreed to the published version of the manuscript.

Funding: This research received no external funding.

Conflicts of Interest: The authors declare no conflict of interest.

\section{References}

1. Vitaku, E.; Smith, D.T.; Njardarson, J.T. Analysis of the Structural Diversity, Substitution Patterns, and Frequency of Nitrogen Heterocycles among U.S. FDA Approved Pharmaceuticals. J. Med. Chem. 2014, 57, 10257-10274. [CrossRef] [PubMed]

2. Heravi, M.M.; Zadsirjan, V. Prescribed drugs containing nitrogen heterocycles: An overview. RSC Adv. 2020, 10, 44247. [CrossRef]

3. Kalogirou, A.S.; Koutentis, P.A. Reactions of Polychlorinated Pyrimidines with DABCO. Molbank 2019, 2019, M1084. [CrossRef]

4. Kalogirou, A.S.; Koutentis, P.A. Synthesis of 2-Cyanopyrimidines. Molbank 2019, 2019, M1086. [CrossRef]

5. Fedorowicz, J.; Gzella, K.; Wiśniewska, P.; Sączewski, J. 2,2'-((1,4-Dimethoxy-1,4-dioxobutane-2,3-diylidene)bis(azanylylidene)) bis(quinoline-3-carboxylic acid). Molbank 2019, 2019, M1093. [CrossRef]

6. Fortunato, M.A.G.; Siopa, F.; Afonso, C.A.M. (1R,4S,5S)-5-((3-Hydroxypropyl)amino)-4-((1-methyl-1H-tetrazol-5-yl)thio)cyclopent2-en-1-ol. Molbank 2021, 2021, M1199. [CrossRef]

7. Manna, F.; Chimenti, F.; Fioravanti, R.; Bolasco, A.; Secci, D.; Chimenti, P.; Ferlini, C.; Scambia, G. Synthesis of some pyrazole derivatives and preliminary investigation of their affinity binding to P-glycoprotein. Bioorg. Med. Chem. Lett. 2005, 15, $4632-4635$. [CrossRef]

8. Kolotova, E.S.; Shtil, A.A.; Novikov, F.N.; Chilov, G.G.; Stroganov, O.V.; Stroilov, V.S.; Zeifman, A.A.; Titov, I.Y.; Sagnou, M.; Alexiou, P. Novel Derivatives of 3,5-Divinyl-pyrazole for Medical Application. WO 2016/190770 A1, 1 December 2016.

9. Matiadis, D.; Mavroidi, B.; Panagiotopoulou, A.; Methenitis, C.; Pelecanou, M.; Sagnou, M. (E)-(1-(4-Ethoxycarbonylphenyl)-5(3,4-dimethoxyphenyl)-3-(3,4-dimethoxystyryl)-2-pyrazoline: Synthesis, Characterization, DNA-Interaction, and Evaluation of Activity Against Drug-Resistant Cell Lines. Molbank 2020, 2020, M1114. [CrossRef]

10. Konstantinova, L.S.; Tolmachev, M.A.; Popov, V.V.; Rakitin, O.A. Ethyl 11a,12-Dihydrobenzo[b]benzo[5,6][1,4]oxazino[2,3e][1,4] oxazine-5a(6H)-carboxylate. Molbank 2020, 2020, M1149. [CrossRef]

11. Tenti, G.; Cores, Á.; Ramos, M.T.; Menéndez, J.C. (E)-3-((2-Fluorophenyl)(hydroxy)methylene)imidazo[1,2-a]pyridin-2(3H)-one. Molbank 2021, 2021, M1212. [CrossRef]

12. Pardali, V.; Katsamakas, S.; Giannakopoulou, E.; Zoidis, G. 1-Methyl-8-phenyl-1,3-diazaspiro[4.5]decane-2,4-dione. Molbank 2021, 2021, M1228. [CrossRef]

13. Vlachou, E.-E.N.; Balalas, T.D.; Hadjipavlou-Litina, D.J.; Litinas, K.E. 4-Amino-2-(p-tolyl)-7H-chromeno[5,6-d]oxazol-7-one. Molbank 2021, 2021, M1237. [CrossRef]

14. Liu, B.; Lim, C.-H.; Miyake, G. Visible Light-Promoted C-S Cross-Coupling via Intermolecular Charge-Transfer. J. Am. Chem. Soc. 2017, 139, 13616-13619. [CrossRef]

15. Tan, B.Y.-H.; Teo, Y.-C. Ligand-free CuI-catalyzed chemoselective S-arylation of 2-mercaptobenzimidazole with aryl iodides. Synlett 2018, 29, 2056-2060. [CrossRef]

16. Ubeid, M.T.; Thabet, H.K.; Abu Shuheil, M.Y. Synthesis of 4-[(1H-Benzimidazol-2-yl)sulfanyl]benzaldehyde and 2-(\{4-[(1HBenzimidazol-2-yl)sulfanyl]phenyl\}methylidene)hydrazine-1-carbothioamide. Molbank 2021, 2021, M1273. [CrossRef]

17. Asquith, C.R.M.; Tizzard, G.J. 6-Bromo-N-(3-(difluoromethyl)phenyl)quinolin-4-amine. Molbank 2020, 2020, M1161. [CrossRef]

18. Husson, J.; Guyard, L. 4'-(5-N-Propylthiophen-2-yl)-2,2':6',2"-terpyridine. Molbank 2021, 2021, M1183. [CrossRef]

19. Banks, S.R.; Yoo, K.M.; Welker, M.E. Synthesis of Polar Aromatic Substituted Terminal Alkynes from Propargyl Amine. Molbank 2021, 2021, M1206. [CrossRef]

20. Halevas, E.; Hatzidimitriou, A.; Mavroidi, B.; Sagnou, M.; Pelecanou, M.; Matiadis, D. Synthesis and Structural Characterization of (E)-4-[(2-Hydroxy-3-methoxybenzylidene)amino]butanoic Acid and Its Novel Cu(II) Complex. Molbank 2021, 2021, M1179. [CrossRef] 
21. Takase, T.; Kainuma, S.; Kanno, T.; Oyama, D. Cis-Bis(2,2'-Azopyridinido)dicarbonylruthenium(II). Molbank 2021, 2021 , M1182. [CrossRef]

22. Aitken, R.A.; Sonecha, D.K.; Slawin, A.M.Z. Homopiperazine (Hexahydro-1,4-diazepine). Molbank 2021, 2021, M1200. [CrossRef] 